\title{
Recently attended masks are less effective
}

\author{
Trafton W. Drew and EdWard K. Vogel \\ University of Oregon, Eugene, Oregon
}

\begin{abstract}
Much work has been done to investigate participants' ability to detect repeated targets, but the present study examined the influence of recently attended stimuli on target masking. Participants performed a target identification task in which the item that masked the target was either a recently attended item or a novel item. When it was identical to a previously attended stimulus, the mask was rendered considerably less effective. We have termed this effect a repeated mask reduction (RMR). This simple manipulation resulted in a large, reliable effect on the efficacy of visual masking in a single-target identification paradigm. In Experiments 1, 2, and 3, we demonstrated the basic effect and noted that the RMR increased as task difficulty increased. Experiments 4 and 5 suggested that attention to the first instance of the mask was crucial to this effect by showing that the magnitude of the RMR was unaffected by repetition of nonmask distractors and that the magnitude of the effect was reduced when less attention was paid to the first instance of the mask.
\end{abstract}

In visual perception, special rules often apply to items that have recently been viewed. Passive perception of the initial presentation of a repeated stimulus can increase the speed and accuracy of later perception of a second presentation of the stimulus, a phenomenon known as repetition priming (see, e.g., Forster \& Davis, 1984). On the other hand, repetition blindness (RB; see, e.g., Kanwisher, 1987) is a phenomenon in which the perception of the second instance of a repeated target is sometimes impaired when the targets are embedded in a rapid serial visual presentation (RSVP) stream. Furthermore, under certain circumstances, the second instance of a stimulus is associated with less BOLD activation in fMRI research (see, e.g., Dehaene et al., 1998; Henson, 2003; Schacter \& Buckner, 1998), and in nonhuman primates, some stimulus-selective neurons in the inferotemporal cortex show reduced firing rates, whereas other neurons in the same area show increased firing rates after stimulus repetition (Miller \& Desimone, 1994). The present set of studies is an attempt to further explore the effect of repeated stimuli on visual perception.

With the notable exception of the Miller and Desimone (1994) study, in which the authors were specifically interested in repetition of nontarget stimuli, one similarity between most studies involving repeated stimuli is that the presence or absence of an effect is measured as a consequence of some behavior toward the second instance of the repeated stimulus. This approach has been successful in advancing the understanding of how repeated stimuli are processed. However, because a judgment is being made about the repeated stimulus in each case, it may be difficult to determine whether the effect is due to a perceptual difference, a higher level influence of memory for the first instance of the stimulus, or response bias. For example, bias against reporting the same target twice appears to have a strong influence on RB, and some have suggested that the $\mathrm{RB}$ deficit may be entirely attributable to response bias in certain versions of the paradigm (Anderson \& Neill, 2002). In the present study, we minimized these response bias issues by repeating nontarget stimuli, and measured the effects of repetition by examining how well these repeated items masked a separate target. Thus, the participants were never required to report the identity of the repeated items, but we could infer the influence of repetition by examining how well these items served as masks.

Target masking is a ubiquitous technique in experimental psychology for regulating the difficulty of a task, yet our understanding of the basic principles of masking is constantly evolving (e.g., Breitmeyer \& Ogmen, 2000; Enns \& Di Lollo, 1997). This article is primarily interested in backward pattern masking: masking that involves spatial superimposition of target and mask contours. Traditional psychophysical explanations of this type of masking often assume that the mask results in a cessation of any target processing once the mask is presented. However, recent developments in the masking literature have suggested that rather than being a strictly low-level process, higher level cognitive processes such as perceptual grouping (see, e.g., Kurylo, 1997), selective attention (Di Lollo, Enns, \& Rensink, 2000), and visual short-term memory consolidation (Vogel, Woodman, \& Luck, 2006) may be disrupted by the presence of a backward mask. In this study, we attempt to extend these findings by examining whether the within-trial context may modulate the efficacy of a mask. Masking effects are most commonly characterized in terms of how the mask relates to the target; the present article examines whether the relationship between attended distractors and the mask will influence the efficacy of the target mask. We take the most extreme 


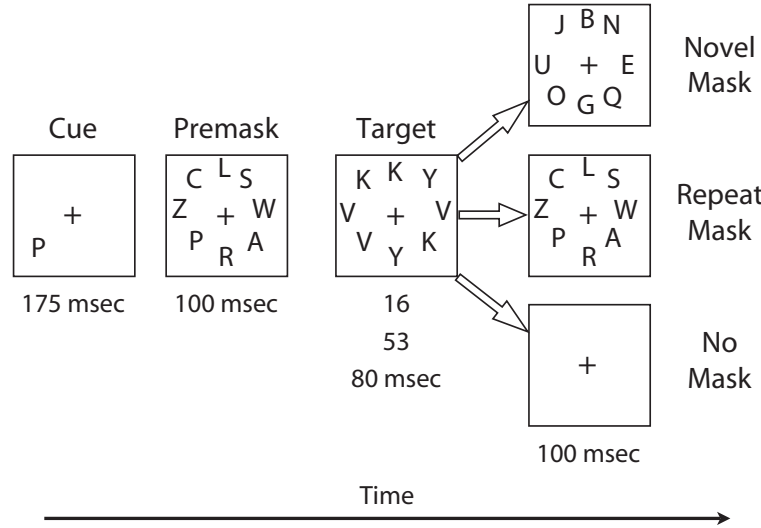

Figure 1. A representation of the Experiment 1 paradigm. Letters are not drawn to scale. The background in the experiment was uniform gray.

case, in which the mask is exactly the same as a previously attended stimulus, and observe that this leads to a greatly reduced masking effect.

The present study was motivated by recent work on masking in the attentional blink $(\mathrm{AB})$. The $\mathrm{AB}$ is the finding that when two targets (T1 and T2) are embedded in an RSVP stream, an observer's ability to detect the second target is severely impaired if it is presented between 200 to $500 \mathrm{msec}$ after the first target (Chun \& Potter, 1995; Raymond, Shapiro, \& Arnell, 1992, 1995). Recently, Drew and Shapiro (2006) found that the AB was reduced when items in the task were repeated twice in a single trial. That is, when $\mathrm{T} 1$ was identical to the item that followed $\mathrm{T} 2$, the $\mathrm{AB}$ was substantially reduced. Thus, the use of a recently attended stimulus as a mask for T2 may have decreased its effectiveness in disrupting target processing. However, it is not currently clear whether this repetition effect is idiosyncratic to dual-task paradigms such as the $\mathrm{AB}$ or whether it reflects a more general effect of repetition on masking. Therefore, in the present study, we sought to extend Drew and Shapiro's finding to a single-target identification paradigm.

\section{EXPERIMENT 1}

In Experiment 1, we sought to provide an initial test of masking repetition effects in a single-target paradigm. Each trial began with a single letter that served as a location cue, informing participants where the target would appear (100\% validity) within a circular array with eight positions (see Figure 1). Following this cue, the remaining positions were filled with distractors (premask array); this was followed by an array of letters including the target (target array). Immediately following the target array was a postmask array, which included letters at each of the eight positions. On repeat mask trials, the letter that followed the target was identical to the initial location cue. On novel mask trials, the letter that masked the target was different from the item that served as a location cue. Because the location cue was indicated using an abrupt object onset in an otherwise empty screen, the first instance of the mask was likely to automatically capture spatial attention (Theeuwes, 1991; Yantis \& Jonides, 1990). Thus, this paradigm allowed us to directly compare the efficacy of masks that have been recently attended with items that have not been recently attended. On the basis of the results of Drew and Shapiro (2006), we expected that repeated masks would be less effective than novel masks, despite the fact that both types of mask draw from the same set of stimuli. We also manipulated task difficulty by varying the duration of the target screen to determine whether the masking effects interacted with target discrimination difficulty.

\section{Method}

Participants. Twelve undergraduate students (5 of them male, average age 19.3 years) from the University of Oregon participated individually in a single 30 -min experimental session for course credit.

Apparatus and Stimuli. In all four experiments reported here, the stimuli were presented on a 15 -in. color monitor driven by a Pentium III computer. Each participant sat roughly $50 \mathrm{~cm}$ from the monitor in a dimly lit room. The stimuli were black letters displayed on a uniform gray screen. In Experiments 1, 2, and 3, each letter was approximately $1.1^{\circ} \times 0.7^{\circ}$ of visual angle. The letters were presented in one of eight locations in a circular array with a radius of $6.8^{\circ}$.

Design and Procedure. The stimuli and procedure are illustrated in Figure 1. Each trial began with a central fixation point and was initiated by the participant. Participant initiation of the trial was followed by a 500 -msec blank interval; then a single capital letter (any letter other than "K," "V," "U," or "Y") was displayed in the target location for $175 \mathrm{msec}$. This was immediately followed by the onset of seven additional distractor letters in a circular display around fixation for $100 \mathrm{msec}$. In this and all subsequent experiments, there was no interstimulus interval. Immediately following the premask array, the target array (a circular array of eight potential targets) followed, and was displayed for 16,53 , or $80 \mathrm{msec}$. The participant's task was to identify whether a "K," "V," or "Y" occurred in the cued location. The backward mask immediately followed and was presented for $100 \mathrm{msec}$. In the repeat mask (RM) condition, this array was identical to the forward mask array, whereas in the novel mask condition, none of the letters were repeated. A blank screen was displayed in the no-mask condition. This resulted in a 3 (target duration) $\times 3$ (mask type) design. The experiment began with 24 practice trials, followed by 270 experimental trials. Participants were instructed to stress accuracy rather than response time.

\section{Results}

Figure 2 displays target discrimination accuracy for the three mask conditions across the three target durations. Mean accuracy was highest (94\%) for the no-mask condition, second highest (73\%) for the RM condition, and lowest $(60 \%)$ for the novel mask condition. In addition, accuracy appeared to increase as duration target increased. This pattern of results was confirmed in an omnibus ANOVA, yielding significant main effects of mask type $[F(2,22)=$ $85.82, p<.001]$ and target duration $[F(2,22)=51.09, p<$ .001]. There was also a significant mask type $\times$ target duration interaction $[F(4,44)=5.69, p<.005]$. Importantly, planned comparisons revealed that performance in the RM condition was significantly higher than in the novel mask condition $[F(1,11)=27.29, p<.001]$. Interestingly, the improvement in the RM condition over the novel mask condition appeared to be primarily evident in the short target duration [16 msec; $t(11)=3.59, p<.05]$ and medium target duration [50 msec; $t(11)=4.56, p<.05]$ condi- 


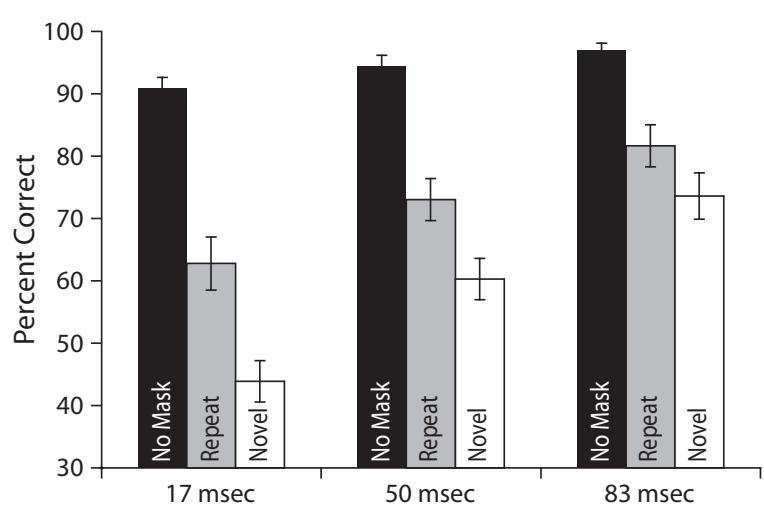

Figure 2. The mean proportion correct of target identification as a function of target duration in Experiment 1. Error bars represent the standard error of the mean. Note that chance performance in this task would be at $33 \%$ correct.

tions; this effect appeared to be considerably smaller at the longest target duration $[t(11)=1.87, p=.09]$.

\section{Discussion}

As is evident in Figure 2, the repeated masks were much (as much as 18\%) less effective in a single-target identification task than were novel masks. That is, within a single trial, a backward mask that had been recently attended was less effective than a novel mask. Performance in the nomask condition was informative here, because it showed that, despite the fact that repeated masks were less effective than novel masks, they still made target identification significantly more difficult than when there was no mask. Thus, repeated masks may be less effective, but they still make the target more difficult to accurately report, even at the shortest target duration.

Furthermore, this effect appears to interact with target duration: As target duration decreases, so does the magnitude of the repeated mask reduction (RMR). When the task was very difficult (17-msec target duration), targets masked by repeated stimuli were much easier to correctly identify $(63 \%$ correct) than were those masked by novel stimuli (44\% correct), but this difference became negligible at the longest target duration. Unfortunately, though, in this experiment, target difficulty was confounded with the period of time between the first and second instances of the mask. Thus, it is unclear whether this aspect of the results was due to the repetition effect being larger when the target was more difficult to perceive or whether it was due to a weakening of the effect as the delay increased.

\section{EXPERIMENT 2}

Unfortunately, because target difficulty was manipulated through exposure duration, an alternative hypothesis is that the repetition effect decreases as the time between the first and second instance of a repeated mask increases. Experiment 2 sought to alleviate this problem by manipulating target difficulty while holding the target duration constant. Squares with either large or small gaps were used to manipulate target difficulty. If target discrimination difficulty is critical in predicting the magnitude of the RMR, there should be a larger effect when the target is more difficult. Conversely, if the difficulty effect found in Experiment 1 was due to the period of time between the first and second instances of the mask, target difficulty should cause a main effect but should not interact with the difference between the repeat and novel conditions.

\section{Method}

Participants. Nineteen undergraduate students ( 7 of them male, average age 19.4 years) from the University of Oregon participated for course credit.

Procedure. The procedure in Experiment 2 was identical to that in Experiment 1, with the following exceptions (see Figure 3). Four, rather than eight, locations around the fixation point were used. The target duration was $50 \mathrm{msec}$ for each trial. Rather than "K," "V," or "Y," the targets in this experiment were squares with a gap in various orientations. The participants' task was to identify the orientation (left, up, or right, but never down) of the gap in the square at the cued location. Target difficulty was manipulated by adjusting the size of the open face of the square. Gap sizes in the two conditions were $0.09^{\circ}$ in the large-gap condition and $0.02^{\circ}$ in the small-gap condition. The design of this experiment was 3 (mask type: repeat, novel, no mask) $\times 2$ (target difficulty: small gap, large gap). The experiment began with 24 practice trials, followed by 192 experimental trials, yielding 32 trials per condition.

\section{Results}

Regardless of mask type, participants were significantly better when there was a large gap in the square $(82.8 \%$ correct) than when there was a small gap (72.5\%), showing that target difficulty was effectively manipulated by gap size $[F(1,18)=40.17, p<.05$; see Figure 4]. Furthermore, performance was better when the first and second masks were repeated, regardless of target difficulty $[F(1,18)=7.13, p<.05]$. Most importantly, there was a significant interaction between mask type and target difficulty $[F(1,18)=4.90, p<.05]$. Planned comparisons revealed that this effect was driven by the fact that performance in the repeated small-gap condition was significantly better than performance in the novel small-gap condition $[t(18)=3.15, p<.05]$, but there was no difference between repeated and novel masks when the gap was large $[t(18)=.56, p>.05]$.

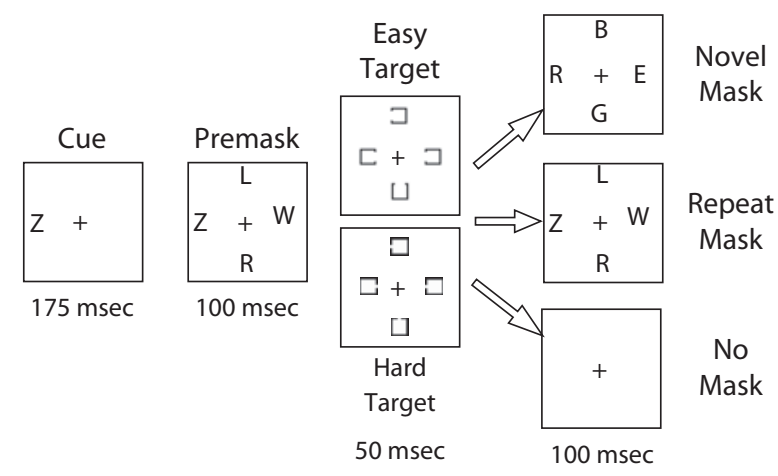

Figure 3. The stimuli and procedure for Experiment 2. 




Figure 4. Performance for Experiment 2. Task performance is plotted as a function of the size of the target gap in the novel, repeat, and no-mask conditions.

\section{Discussion}

It appears that target discrimination difficulty and RMR do indeed interact: When target identification is easier, there is no difference between performance in the repeated and novel conditions, but when the task is more difficult, performance is significantly better in the RM condition. Furthermore, this lack of difference is not simply due to a ceiling effect, because subjects were still well below no-mask performance. Although this experiment alone is not sufficient to rule out the time between the first and second instances of the mask as a factor in the RMR, it provides strong evidence that target difficulty does play a role. It seems likely that target difficulty and time between mask repetition interacted in the first experiment, whereas in the present experiment, the repeated mask had no effect in the easy condition. When the target is easy to perceive due to either perceptual manipulation (e.g., gap size) or increased target duration, repeated masks have little to no advantage over novel masks.

\section{EXPERIMENT 3}

An alternative explanation to the present set of results is that repeated masks, rather than serving as less effective masks, may serve as a retrieval cue that reduces the uncertainty of the target location. For example, if the participant were able to remember the cue identity, searching the target mask screen would allow the participant to deduce the target location, but only in the RM condition. Thus, the performance advantage in the RM condition could simply be the result of decreased uncertainty about the target location (see, e.g., Shiu \& Pashler, 1994). To test this alternative hypothesis, we manipulated the availability of a retrieval cue across the novel and RM conditions. In the two single-mask conditions, the target mask screen was a single item at the target location. The single mask served as a retrieval cue because it was always at the target location and thereby minimized any location uncertainty. By contrast, the two filled-mask conditions were identical to those in our previous experiments, in which masks were presented at all locations. If the retrieval cue plays a role in the repeated mask effect found in previous experiments, we would expect an interac- tion between retrieval cue type (present or absent) and mask type (repeated or novel). The retrieval cue account predicts improved performance when target location uncertainty is reduced. Thus, performance on novel mask trials should be better when there is a single retrieval cue, thereby reducing or eliminating the repeated mask effect in this condition.

\section{Method}

Participants. A total of 22 undergraduate students ( 8 of them males, average age 20 years) from the University of Oregon participated for course credit.

Design and Procedure. The stimuli and procedure are illustrated in Figure 5. This experiment was identical to Experiment 2, with the following exceptions. The target screen was displayed for $34 \mathrm{msec}$ in each trial and contained four potential targets. On half of the masked trials, the target mask screen contained a single letter. This letter served as a retrieval cue because it was always at the target location. In contrast, the filled RM and filled novel mask conditions contained four letters and therefore no explicit target location cue. Target mask duration was $200 \mathrm{msec}$. The experiment began with 30 practice trials, followed by 175 experimental trials. There were five conditions (no mask, filled repeat mask, filled novel mask, single repeat mask, and single novel mask), yielding 35 trials in each condition.

\section{Results}

This was a difficult task (see Figure 6), with participants well off ceiling even in the no-mask condition ( $75 \%$ correct). Primary analyses were done by comparing the filled-mask conditions with the single-mask conditions as a function of mask type (repeated or novel), resulting in a $2 \times 2$ design. There was a significant effect of mask type $[F(1,21)=$ $12.49, p>.01$ ] but no main effect of retrieval cue type, and the interaction did not approach significance (both $F_{\mathbf{S}}>1$ ). Planned comparisons revealed that there was a repeat mask effect (repeat mask performance $>$ novel mask performance) in both the presence $[t(21)=2.92, p>.05]$ and absence $[t(21)=2.84, p>.05]$ of an explicit retrieval cue.

\section{Discussion}

These results provide strong evidence against the retrieval cue explanation for our previous results. There was a strong main effect of mask type that did not interact with the presence or absence of a single retrieval cue in the target's location. It appears that RMR is due to something special

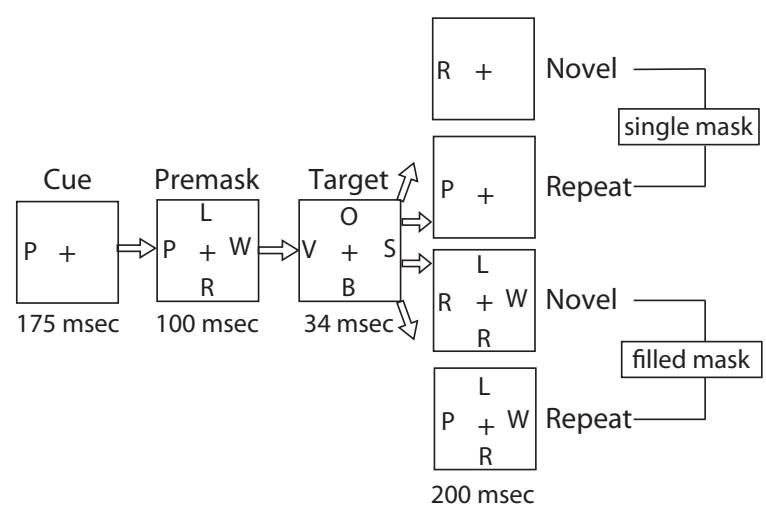

Figure 5. The stimuli and procedure for Experiment 3. 


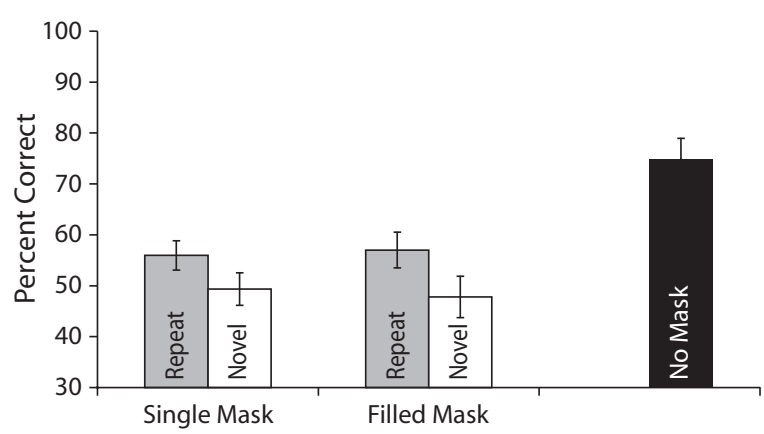

Figure 6. Performance for Experiment 3. Single-mask conditions contained an explicit cue for the target location, whereas the filled-mask conditions did not.

about an attended mask repetition rather than to the disambiguation of the target location. Eliminating the irrelevant items in the target mask screen did not affect the difficulty of the task, in spite of the fact that duration was twice as long $(200 \mathrm{msec})$ as in any of the previous experiments. It therefore appears unlikely that repeated masks were used as retrieval cues in any of our experiments. In light of the present results, it seems that the task was difficult due to target identity uncertainty rather than location uncertainty.

\section{EXPERIMENT 4}

In both Experiment 1 and Experiment 2, all of the items in the forward and backward mask arrays were held constant in the RM condition. This opens the possibility for an alternative explanation for our results. Perhaps repeated masks were less effective in these experiments because the participant perceived the target array as flashing in front of a stationary background (i.e., the forward and backward mask arrays). However, in the novel condition, there could be no perception of a stagnant background because the forward and backward mask arrays were completely different. If this global background hypothesis is correct, then the magnitude of the RMR should be closely related to the global similarity of forward and backward arrays. We tested this hypothesis by replicating Experiment 1 with an additional condition (see Figure 7). In this condition, the letter in the target position was identical in the forward and backward mask arrays, but none of the other letters were held constant. This condition (repeat mask only) was directly contrasted with the repeat condition from the previous experiment (repeat everything). If the global background hypothesis is correct, performance in the repeat-mask-only condition should be worse than in the repeat-everything condition, and should approach performance in the novel condition. On the other hand, if repetition of local information is necessary and sufficient for the RMR, the two repeat conditions should be equivalent.

\section{Method}

Participants. Seventeen participants (4 of them male, average age 19.8 years) from the same participant pool as was used in Experiment 1 took part.
Design and Procedure. Experiment 4 was identical to Experiment 1, with two exceptions. Target duration was held constant at $32 \mathrm{msec}$, and the repeat-mask-only condition (described above) was added to the experimental design. After 52 practice trials, participants completed 200 trials, resulting in 50 trials in each of the four conditions (i.e., no mask, novel mask, repeat everything, repeat mask only).

\section{Results}

The results of Experiment 4 are shown in Figure 8. Performance in the no-mask condition was near ceiling (total accuracy 97\%) for all participants and was therefore excluded from subsequent statistical analyses. There was a strong effect of mask type between the remaining three groups: repeat everything, repeat mask only, and novel $[F(2,32)=31.59, p<.001]$. Planned comparisons showed that there were no significant differences between the repeat-everything $(67.8 \%)$ and the repeat-mask-only $(69.1 \%)$ conditions $[t(16)=.78, p=$ n.s. $]$. However, performance in both of those conditions was significantly higher than in the novel condition $(56.5 \%)[t(16)=7.75$, $p<.05 ; t(16)=5.87, p<.05$, respectively $]$.

\section{Discussion}

Performance in the repeat-everything and repeat-maskonly conditions was statistically identical. Performance in both conditions was significantly better than performance in the novel mask condition, providing an average of an $11 \%$ advantage in accurately reporting the target. It appears that the stagnant global mask hypothesis cannot account for the benefit of repeated masks: Repetition of local information alone resulted in an RMR that was equivalent to that observed with the repetition of the entire array. Thus, repetition of irrelevant distractors does not appear to influence this masking effect. This suggests that focal attention to the item that masks the target may be critical to observing RMR. Consequently, repetition in an attended location results in reduced masking, whereas repetition in unattended locations appears to have no influence at all.

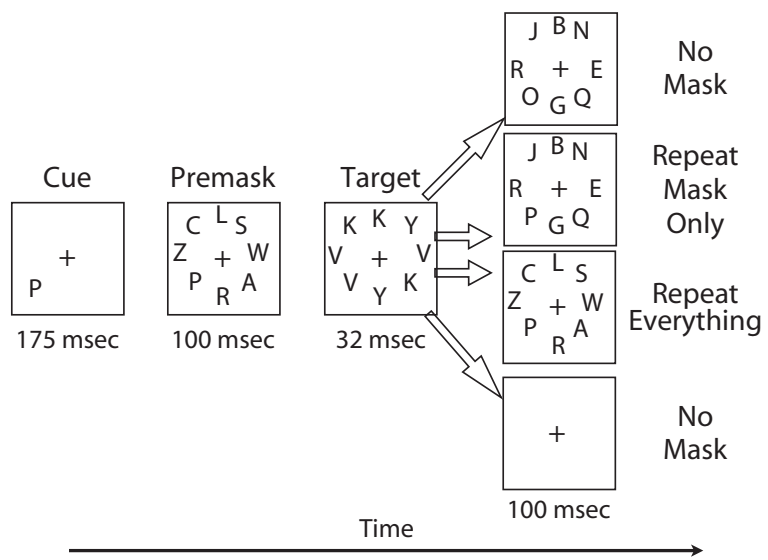

Figure 7. The stimuli and procedure for Experiment 4. 


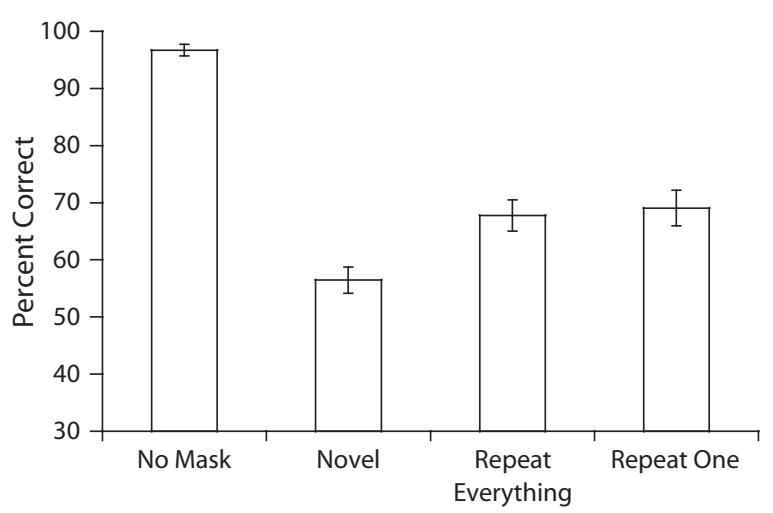

Figure 8. The mean percentage correct as a function of mask type for Experiment 4.

\section{EXPERIMENT 5}

The results of Experiment 4 suggest that repetition of unattended objects has no effect on RMR. Therefore, it is plausible that focused attention to the item that has been repeated is critical for observing a repetition effect. In the first three experiments, the item that masked the target was always attended because it cued the correct location of the target with $100 \%$ certainty. In contrast, an alternative account of the RMR is that it is an automatic effect that can occur even when attention is not directly focused on the premask. Experiment 5 sought to test these hypotheses by manipulating the number of location cues. When there was only one location cue, the target was always in that position (as in all previous experiments). Critically, in the two-cue condition, the target was equally likely to occur in either of the cued positions (see Figure 9). In single-cue trials, we assume that participants attend the first instance of the mask. However, in the two-cue trials, attention is likely to be divided or spread across the two locations; thus, there should be less attention allocated to each of the premasks. If spatial attention is not necessary for RMR, the location specificity of the cue should not influence the magnitude of the repetition effect. However, if focused attention to the premask is necessary, the magnitude of the RMR should be larger in the one-cue condition than in the two-cue condition.

\section{Method}

Participants. Sixteen undergraduate students (4 of them males, average age 19 years) from the University of Oregon participated for course credit.

Apparatus and Stimuli. Each letter was approximately $1.6^{\circ} \times$ $.7^{\circ}$ of visual angle; the letters appeared $9.1^{\circ}$ above, below, right, or left of fixation.

Design and Procedure. The stimuli and procedure are illustrated in Figure 9. This experiment was identical to Experiment 2, with the following exceptions. Because of the cuing manipulation, the target array contained only the letter "K," "V," or "Y." On half of the trials, the target was cued with two letters on either side of the fixation (left and right or above and below, but never left and above), and participants were instructed that the target would appear equally in either of the cued locations. The experiment began with 24 practice trials, followed by 192 experimental trials. Given the 3 (mask type: repeat, novel, or no mask) $\times 2$ (cue type: 2 or 1 ) design, this resulted in 32 trials in each condition.

\section{Results}

Performance in the no-mask condition was very high, regardless of cue type $(91.8 \%$ correct in the one-cue condition and $88.9 \%$ correct in the two-cue condition), and was therefore not entered into further analyses. Accuracy was higher in the one-cue $(70.5 \%)$ than in the twocue $(56.7 \%)$ condition, resulting in a main effect of cue type $[F(1,15)=68.92, p<.001$; see Figure 10$]$. There was also a main effect of mask type $[F(1,15)=28.47$, $p<.001$ ], with performance generally better in the RM condition. Critically, there was a significant interaction between cue and mask type $[F(1,15)=5.14, p<.05]$. Planned comparisons revealed that performance was significantly better in the RM condition when there was one cue $[t(15)=4.7, p<.001]$, but there was no difference between repeated and novel masks when two locations were cued $[t(15)=1.8, p=$ n.s. $]$.

\section{Discussion}

These results suggest that repeated masks that have been recently attended are less effective than novel masks. Given the significant effect of cue type, we can infer that participants were most likely using the cue to focus spatial attention toward the target location in the task. This suggests that spatial attention was focused on the single premask in the one-cue condition, but was much less likely to be focused on the correct location in the two-cue condition. Thus, Experiment 5 shows that the RMR is essentially eliminated when less attention is paid to the first mask. However, when the forward mask is attended (in the onecue condition), there is a large benefit of mask repetition ( $15 \%$ improvement in accuracy). This suggests that RMR is not an automatic process brought on by any repeated stimuli. Rather, it appears that in order for a repeated mask to be less effective, it is necessary for the first instance of the mask to have spatial attention directly focused on it.

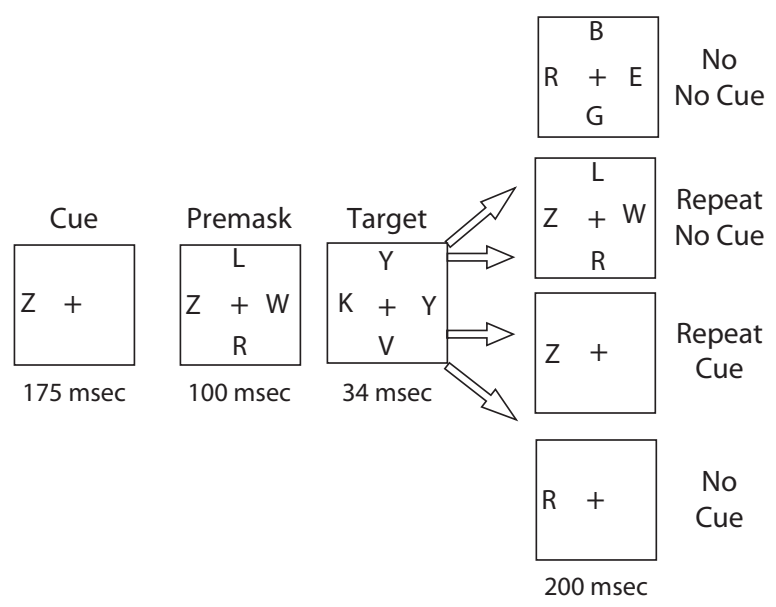

Figure 9. The stimuli and procedure for Experiment 5. 


\section{GENERAL DISCUSSION}

Recent work on the $\mathrm{AB}$ has found that when either the first-target or second-target mask was a repetition of a previously attended letter, the magnitude of the $A B$ was reduced (Drew \& Shapiro, 2006). The present experiments demonstrate that this repetition effect is not dependent on dual-task paradigms such as the $\mathrm{AB}$, but appears to be a more general property of masking. The present study demonstrated that attention to the first instance of the mask is critical for observing an RMR. Furthermore, when attention is directed to local properties, global repetition is irrelevant within a single trial. Finally, RMR appears to interact with task difficulty; increased task difficulty results in a larger masking reduction.

Masking effects are typically considered in terms of the mask's relationship, both perceptually and temporally, with the target. However, the present results suggest that previously attended items may greatly influence the efficacy of a backward mask. The average reduction of masking effects through repetition in this set of experiments was $10 \%-15 \%$. Although it appears to interact with task difficulty, RMR appears to be based primarily on the relation between an attended distractor and the item that masks the target. The fact that repeated stimuli serve as less effective masks suggests that representations of attended stimuli are stored by the visual system and that this memory of a recent stimulus may influence the processing of a subsequent presentation of an identical item.

The results provide more evidence toward the already well-substantiated claim that, within the context of the visual system, repeated stimuli are special (Henson, 2003; Kanwisher, 1987; Kanwisher \& Potter, 1990; Miller \& Desimone, 1994). What makes the present set of experiments unique in this literature is the approach taken to make this point. Rather than evaluating how repeated stimuli are processed on the basis of report of a repeated stimulus, we are indirectly inferring the effect of repetition by analyzing the ability of repeated stimuli to mask unrelated targets. This approach has several advantages over the more direct approach. In each of the experiments reported, presence or absence of a repeated stimulus was completely irrelevant to the observer's task. Most of our participants were not aware that any letters were repeated in the experiment. In contrast, in the majority of studies that examine the effect of repeated stimuli, participants must be explicitly warned that targets will be repeated frequently. One common criticism of the RB literature is that response bias could help explain the effect (Anderson \& Neill, 2002). Although a great deal of work has been done to demonstrate that response bias does not account for the majority of the effect size in many RB experiments, the present approach is able to bypass this question because participants never respond to the repeated stimulus (Fagot \& Pashler, 1995; Johnston, Hochhaus, \& Ruthruff, 2002; Whittlesea \& Wai, 1997).

\section{Possible Mechanisms}

Our present results appear to be generally consistent with established models of masking, but may require some

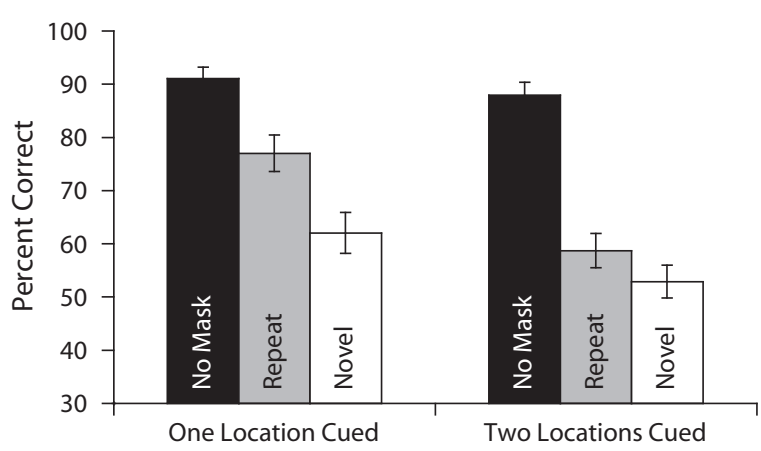

Figure 10. Performance in Experiment 5. Data are plotted for the novel, repeat, and no-mask conditions as a function of the number of locations cued prior to target presentation.

modest modifications to account for the repetition effect. When visual stimuli are presented, there is an initial transient neuronal response, sometimes called an ON transient, followed by a sustained neuronal response that is thought to reflect stimulus processing and identification (Breitmeyer $\&$ Ganz, 1976). The ON transient is generally considered to act as an event detector, whereas sustained channel activity is necessary to encode enough information about the target for conscious report. Masking effects are often attributed to the transient response from a mask's interrupting the sustained channel's processing of a previously presented target. It is our contention that a repeated mask is less effective at interrupting target processing, thereby allowing more of the sustained signal to be processed and resulting in reduced masking efficacy. It seems plausible that recent transient activation due to a specific visual input may lead to a reduced transient response the second time an identical stimulus is attended. We believe that the RMR may occur as a consequence of a reduced transient response to the second instance of a repeated stimulus. In our paradigm, each stimulus should elicit both a transient and a sustained response, with the transient response of the backward mask interrupting the sustained response of the target. This interpretation predicts that as the time between the first and second instances of the mask increases, the effect of repetition should decrease (Philips \& Singer, 1974). Experiment 1 provides evidence in favor of this view, although Experiment 2 shows that target difficulty may interact with the time between ON transient activation. Furthermore, this interpretation suggests that an RMR may occur even when the first instance of the mask is embedded in a stream of attended distractors. Accordingly, Dux, Coltheart, and Harris (2006) recently found that the AB is reduced when the first target is immediately preceded and followed by the same distractor. However, the effect did not generalize across letter case. It seems that the repetition of contours, rather than an abstract representation of type, is responsible for the reduction in masking.

Marcel (1983) postulated that, rather than interfering with target analysis, in some cases masking occurs because the target and the mask are grouped together. Linking the backward mask to the forward mask may therefore encourage the perception of the target and the mask 
as two, rather than three, distinct items. There appears to be a plausible object level component to both the RMR and to object substitution masking (OSM; Di Lollo et al., 2000; Enns \& Di Lollo, 1997). OSM occurs when a sparse mask is presented simultaneously with the target, and the target offsets first, leaving the mask alone. Under certain circumstances, this is an effective method of masking despite very little contour overlap. OSM is reduced when the target and mask are made to appear as separate objects (Moore \& Lleras, 2005), whereas manipulations that would increase the perception of the item before and after the target as being a single object (such as decreased target duration and attention to the first instance of the mask) decrease masking efficacy in the RMR effect as well. Although we consider decreased $\mathrm{ON}$ transient response to be the mechanism of the RMR, OSM is generally thought to be due to a mismatch between the reentrant signal and the ongoing lower level activity (Enns \& Di Lollo, 2000). It is not yet clear whether a similar mechanism is responsible for both processes, but it is known that OSM is still effective when the mask and target are in different locations. In future experiments, we hope to better understand the RMR effect by comparing it with better understood masking paradigms such as OSM and metacontrast masking.

\section{Summary}

Using a simple target identification paradigm, we have found strong evidence that the second instance of a repeated stimulus serves as a poor backward pattern mask. It appears that, even when irrelevant to the task and not a member of the target set, repeated stimuli are processed differently than those that are not repeated. The primary purpose of the present set of experiments was to map out some of the initial parameters of this effect. Provided that the first instance of the mask is attended, this paradigm appears to provide large, robust, and easily replicable repetition effects. Further work will be necessary to better understand some additional basic parameters, such as the time course of the repetition effect, as well as the specificity of the overlap between the first and second instances of the mask.

\section{AUTHOR NOTE}

This study was supported by grants from the National Science Foundation and the Oregon Medical Research Foundation. Correspondence concerning this article should be addressed to E. K. Vogel, Department of Psychology, 1227 University of Oregon, Eugene, OR 97403-1227 (e-mail: vogel@uoregon.edu).

\section{REFERENCES}

Anderson, C. J., \& Neill, W. T. (2002). Two Bs or not two Bs? A signal detection theory analysis of repetition blindness in a counting task. Perception \& Psychophysics, 64, 732-740.

BreitMEYer, B. G., \& GANZ, L. (1976). Implications for sustained and transient channels for theories of visual pattern masking, saccadic suppression and information processing. Psychological Review, 81, 1-36.

Breitmeyer, B. G., \& Ogmen, H. (2000). Recent models and findings in visual backward masking: A comparison, review, and update. Perception \& Psychophysics, 62, 1572-1595.

Chun, M. M., \& PotTer, M. C. (1995). A two-stage model for multiple target detection in rapid serial visual presentation. Journal of Experimental Psychology: Human Perception \& Performance, 21, 109-127.

Dehaene, S., Naccache, L., Le Clec, H. G., Koechlin, E., Muel-
Ler, M., Dehaene-Lambertz, G., et AL. (1998). Imaging unconscious semantic priming. Nature, 395, 597-600.

Di Lollo, V., Enns, J. T., \& Rensink, R. A. (2000). Competition for consciousness among visual events: The psychophysics of reentrant visual processes. Journal of Experimental Psychology: General, 129, 481-507.

Drew, T., \& ShapIRo, K. (2006). Conceptual masking in the attentional blink. Visual Cognition, 13, 513-528.

Dux, P. E., Coltheart, V., \& Harris, I. M. (2006). On the fate of distractor stimuli in rapid serial visual presentation. Cognition, 99, 355-382.

EnNs, J., \& Di LolLo, V. (1997). Object-substitution: A new form of masking in unattended visual locations. Psychological Science, $\mathbf{8}$, 135-139.

EnNs, J., \& Di LoLLo, V. (2000). What's new in visual masking? Trends in Cognitive Sciences, 4, 345-352.

Fagot, C., \& Pashler, H. (1995). Repetition blindness: Perception or memory failure? Journal of Experimental Psychology: Human Perception \& Performance, 21, 275-292.

Forster, K. I., \& DaVIS, C. (1984). Repetition priming and frequency attenuation in lexical access. Journal of Experimental Psychology. Leaning, Memory, \& Cognition, 10, 680-698.

Henson, R. N. A. (2003). Neuroimaging studies of priming. Progress in Neurobiology, 70, 53-81.

Johnston, J. C., Hochhaus, L., \& Ruthruff, E. (2002). Repetition blindness has a perceptual locus: Evidence from online processing of targets in RSVP streams. Journal of Experimental Psychology: Human Perception \& Performance, 28, 477-489.

KANWISHER, N. G. (1987). Repetition blindness: Type recognition without token individuation. Cognition, 27, 117-143

Kanwisher, N. G., \& Potter, M. C. (1990). Repetition blindness: Levels of processing. Journal of Experimental Psychology: Human Perception \& Performance, 16, 30-47.

Kurylo, D. D. (1997). Time course of perceptual grouping. Perception \& Psychophysics, 59, 142-147.

Marcel, A. J. (1983). Conscious and unconscious perception: Experiments on visual masking and word recognition. Cognitive Psychology, 19, 197-237.

Miller, E. K., \& Desimone, R. (1994). Parallel neuronal mechanisms for short-term memory. Science, 263, 520-523.

Moore, C. M., \& Lleras, A. (2005). On the role of representations in substitution masking. Journal of Experimental Psychology: Human Perception \& Performance, 31, 1171-1180.

Philips, W. A., \& Singer, W. (1974). Function and interaction of ON and OFF transients in vision 1. Psychophysics. Experimental Brain Research, 19, 493-506.

Raymond, J., Shapiro, K., \& ARnell, K. (1992). Temporary suppression of visual processing in an RSVP task: An attentional blink? Journal of Experimental Psychology: Human Perception \& Performance, 18, 849-860.

Raymond, J., Shapiro, K., \& ARNell, K. (1995). Similarity determines the attentional blink. Journal of Experimental Psychology: Human Perception \& Performance, 21, 653-662.

Schacter, D. L., \& Buckner, R. L. (1998). Priming and the brain. Neuron, 20, 185-195.

SHIU, L., \& PASHLER, H. (1994). Negligible effect of spatial precuing on identification of single digits. Journal of Experimental Psychology: Human Perception \& Performance, 20, 1037-1054.

THEeuwes, J. (1991). Exogenous and endogenous control of attention: The effect of visual onsets and offsets. Perception \& Psychophysics, 49, 83-90

Vogel, E. K., Woodman, G. F., \& Luck, S. J. (2006). The time course of consolidation in visual working memory. Journal of Experimental Psychology: Human Perception \& Performance, 32, 1436-1451.

Whittlesea, B. W. A., \& WAI, K. H. (1997). Reverse "repetition blindness" and release from "repetition blindness": Constructive variations on the "repetition blindness" effect. Psychological Research, 60, 173-182

Yantis, S., \& Jonides, J. (1984). Abrupt visual onsets and selective attention: Evidence from visual search. Journal of Experimental Psychology: Human Perception \& Performance, 10, 601-621.

(Manuscript received September 27, 2006; revision accepted for publication June 26, 2007.) 